\title{
NCCN Distress Thermometer and Problem List for Patients Version 2.2016
}

National Cancer Institute

\section{Source}

National Cancer Institute. NCCN Distress Thermometer and Problem List for Patients

Version 2.2016. NCI Thesaurus. Code C132492.

A screening tool developed by the National Comprehensive Cancer Network (NCCN)

Distress Management Panel used to identify patient distress coming from any source and patient problems in five categories. 